\title{
A frame for a computer aided inspection planning system
}

\author{
Polini Wilma $^{1 *}$, Moroni Giovanni ${ }^{2}$ \\ ${ }^{1}$ Dipartimento di Ingegneria Civile e Meccanica, Università di Cassino e del Lazio Meridionale, Cassino, 03043 Cassino, Italy \\ ${ }^{2}$ Dipartimento di Meccanica, Politecnico di Milano, 20133, Italy \\ *Corresponding authorE-mail: polini@unicas.it
}

Copyright () 2015 Polini Wilma, Moroni Giovanni. This is an open access article distributed under the Creative Commons Attribution License, which permits unrestricted use, distribution, and reproduction in any medium, provided the original work is properly cited.

\begin{abstract}
Coordinate Measuring Machine (CMM) inspection planning is an activity performed by well-trained operators, but different measurement techniques, using the same data analysis algorithms yield in different measurement results. This is a well-recognized source of uncertainty in coordinate measurement. A CMM, provided with an automatic inspection planning (CAIP) system, permits to implement more accurate and efficient operating procedures and to fit higher quality assurance standards and tighter production timings.

In this paper we present a frame of a CAIP system, able to deal with almost all the decisional stages of CMM inspection. Moreover, original approaches have been developed and presented in inspection feature selection, part set-up, probe configuration, and path planning.
\end{abstract}

Keywords: Coordinate Measuring Machines; Inspection; Probes; Tolerance Analysis.

\section{Introduction}

The growing request from industry of both a concurrent-integrated manufacturing environment and a certified production causes inspection to have a key role to reach manufacturing reliability and safety. In fact, inspection is a quality assurance tool, which provides feedback on manufacturing processes, and a total quality component, which has to be considered an integral part of the design process.

Many different mechanical products are subject to dimensional or geometrical inspection by measuring devices integrated in the production system. Coordinate measuring machines (CMMs) plays the pivotal role, since they are able to control almost all the dimensional and geometric tolerances on very different parts.

CMM inspection planning is an activity performed by well-trained operators who generally use one of the following programming techniques: teach-in; direct; computer aided. All these methods permit to build an inspection plan but don't consider a fundamental problem in using CMM: different measurement techniques, using the same data analysis algorithms, yield in different measurements results. This is a well recognized source of uncertainty in coordinate measurement [1].

A CMM, provided with an automatic inspection planning (CAIP) system, permits to implement more accurate and efficient operating procedures, and to fit higher quality assurance standards and tighter production timings.

A computer aided inspection planning system aims to reduce the measurement time and uncertainty. In fact it allows to automatically plan all the operations concerning with CMM inspection of mechanical parts by means of optimization techniques. It gets information from a geometric model representation of the part, enriched with tolerance specifications, and makes decision without human intervention, in order to generate a complete CMM part program. The main decisions concern the selection of the features to inspect, the sampling strategies, the probe configuration, the fixture design and the probe path generation.

Many attempts to achieve the goal of automated inspection planning have been reported in literature. A complete state of the art of the early works is presented in [2]. Examples of researches dealing with the development of complete CAIP systems may be reported in [3]-[9]. An updated review of the researches on Computer Aided Inspection Planning of the past 25-years is shown in [10], where some unsolved issues are underlined. 
More recent works represent a first attempt to integrate computer aided inspection with CAPP/CAM for improving the machining process and controlling machining quality [11]-[12]. They are developed for free form surfaces.

The inspection process planning has been mainly carried out in isolation from machining process planning. The proposed solutions did not integrate completely inspection in the product development chain. The systems of the literature did not try to give a feed-back to the process. The aim to overcome those limits is the basis to develop the present work.

\section{A frame for CAIP}

In order to increase inspection efficiency and effectiveness a Computer Aided Inspection Planning (CAIP) system, linking the design stage with co-ordinate measuring machines (CMMs), would allow to automatically plan all the operations concerning with CMM dimensional inspection of a mechanical work-piece since conceptual design. The system should get information from a work-piece CAD representation and make decision without human intervention in order to generate a DMIS part program.

To develop inspection knowledge and algorithms, an analysis of the human based inspection process has been carried out and some procedures for inspection have been identified: interpreting the part geometry and its tolerance specifications; determining inspection requirements for the part; selecting inspection devices and sensors; determining a detailed inspection sequence; conducting the inspection according to the plan.

All these steps must be automated in order to build a CAIP system. In particular, a CAIP system must have the modules shown in Figure 1 that represent the main decisions of a human inspection process.

The Inspection Feature Selection module recognizes, starting from a solid model, the form feature to inspect, i.e. the form feature to which dimensional or geometrical tolerances are applied. A form feature is a component of the real part which binds the accessible directions and the dimensional constraints of a touch trigger probe able to inspect it. A form feature is always a subset of the boundary of the solid model representing a part. Therefore, the form features identified in a part constitute the base to define the geometrical constraints for probe configuration by means of the accessibility analysis.

The Sampling Strategy make decisions about the number and the distribution of the measuring points on each inspection feature. On one hand, the machine probes the part surface point-wise and economic constraints force the set of measured points to be small. On the other hand, geometric errors, as defined by tolerancing standards [13], depend heavily on extreme values of the form deviations over the related part features. For example, straightness is defined as the minimum distance between two parallel lines enclosing the actual feature (Minimum Zone criterion). Hence few points, the most "outward" and "inward" points, have the highest leverage on the straightness error whereas all the others have none, as shown in Figure 2.

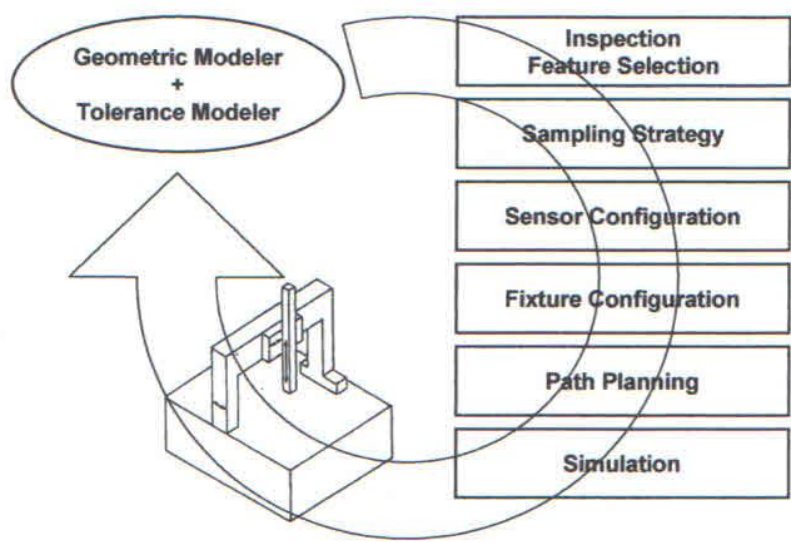

Fig. 1: A CAIP System.

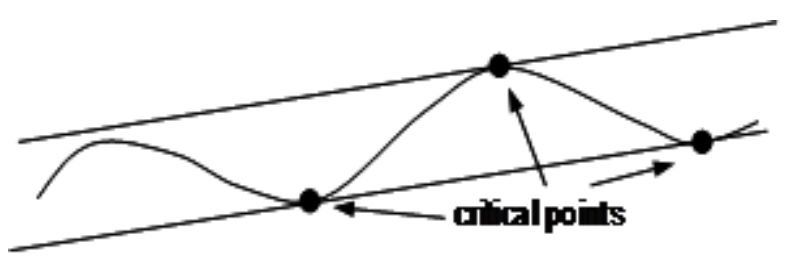

Fig. 2: Straightness Error of the Profile Is Determined by Only Three Critical Points.

Once the system has distributed the measuring points on the part, according to an appropriate sampling strategy, the Sensor Configuration module defines the orientation, dimensions and structure of the touch probes to use. In the same 
time, the Fixture configuration module selects how to orient the workpiece inside the CMM volume and, therefore, it determines the fixtures needed to hold the part in a given spatial configuration (position and orientation) relative to the machine reference frame. The fixture and probe design are related. In fact the choice of a specific part orientation may imply to increase the probes number, the probe changing time and then the inspection time; on the contrary, a set of different part fixtures may require a unique probe configuration, but a greater inspection time connected with the part set-up changing. Therefore, it is needed to find out the optimal trade-off between probe configuration and fixture design with constraints related to the inspection time and quality.

The Path Planning defines the probe path by connecting all the measuring points with a collision free trajectory. It has an impact in improving inspection efficiency and throughput. The main problem in the path planning for a CMM is that it is almost impossible to have a priori knowledge related to the optimal path connecting each couple of points. In fact, given $\mathrm{N}$ measuring points it means to compute $\mathrm{N}(\mathrm{N}-1) / 2$ collision free trajectories. If we consider for example 100 measuring points, it means to compute 4950 paths: a fast algorithm, that takes $1 \mathrm{~s}$ for each path, will need approximately 1 hour and 20 minutes. This is not acceptable in a simultaneous design approach, where the solutions have to be on the designer's finger tips. Therefore, the problem is solved by finding a promising visiting sequence and, then, calculating a sub-optimal collision free trajectory for the sequence.

Finally, the Simulation module shows on PC the probe path by verifying the presence of collisions.

In this paper, a complete frame of a CAIP system has been presented; it is able to deal with almost all the decisional stages of CMM inspection. Particularly, we discuss original approaches in inspection feature selection (Section 3), part set-up and probe configuration (Section 4), and path planning (Section 5). The well-known ANSI/ANC101 benchmark and a second part (called CADME) will be considered as case studies, they are shown in Figure 3.

(A)

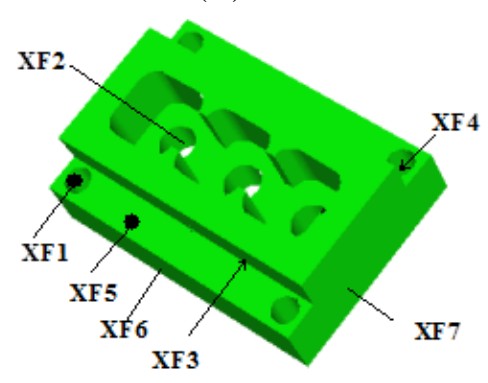

(B)

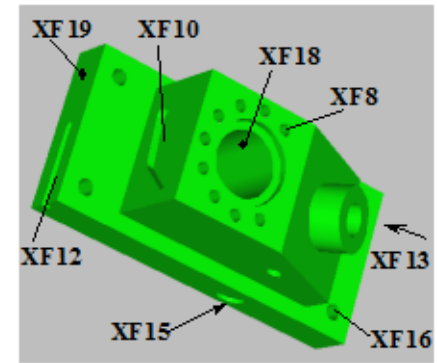

(C)

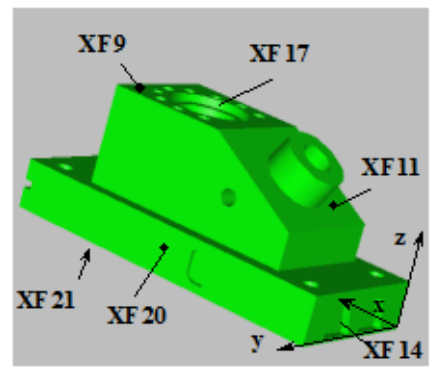

Fig. 3: The Considered Case Studies: A) CADME; B, C) ANSI/ANC 101.

\section{Feature selection}

An Inspection Feature (IF) can be informally defined as the informative content of a tolerance indication together with the technological information necessary to plan its inspection and evaluation.

The number of IFs is, therefore, equal to the number of tolerances in a part drawing. A possible taxonomy of the tolerances has been built up following an appropriate reclassification of the tolerances according to ASME and ISO geometrical product specifications. An IF definition relies on two kind of information: geometric and topologic (extracted from a geometric representation of the part); technological (extracted from a PDM system).

The above ideas result in an IF model where information is distributed on a three level structure [14]. The first level (Inspection Feature level) refers to one or more abstract geometric features (Inspection Form Feature level), such as axes, middle planes, or edges, each knowing how to retrieve the related information through the part faces (Extended Face level). The IF-IFF-XF structure is directly extracted from the tolerance modeler discussed in [14]. An example of such a structure for the ANC101 benchmark is shown in Figure 4.

Starting from such a data structure, an inspection cycle ought to be able to evaluate all the Inspection Features of the part with minimal loss of information due to the uncertainty inherent in the gauging process. Making the hypothesis that the inspection uncertainty is inversely proportional to the number of measuring points, it will be better to be redundant with such number to increase the accuracy of inspection. External constraints, such as production cycle time $\mathrm{T}_{\mathrm{c}}$, bound the set of feasible inspection plans.

The feature selection problem consists in defining an inspection plan which respects such constraints and maximizes the number of IF inspected and the accuracy of the inspection. Therefore, the planner tries to build plans for a fixed set of Inspection Features, optimizing the surface points distribution, the probe selection, and the probe path, while an IF selection algorithm, driven by priority criteria and cycle time estimation, prunes the feature list and diminishes the number of measuring points so to respect the constraints. 


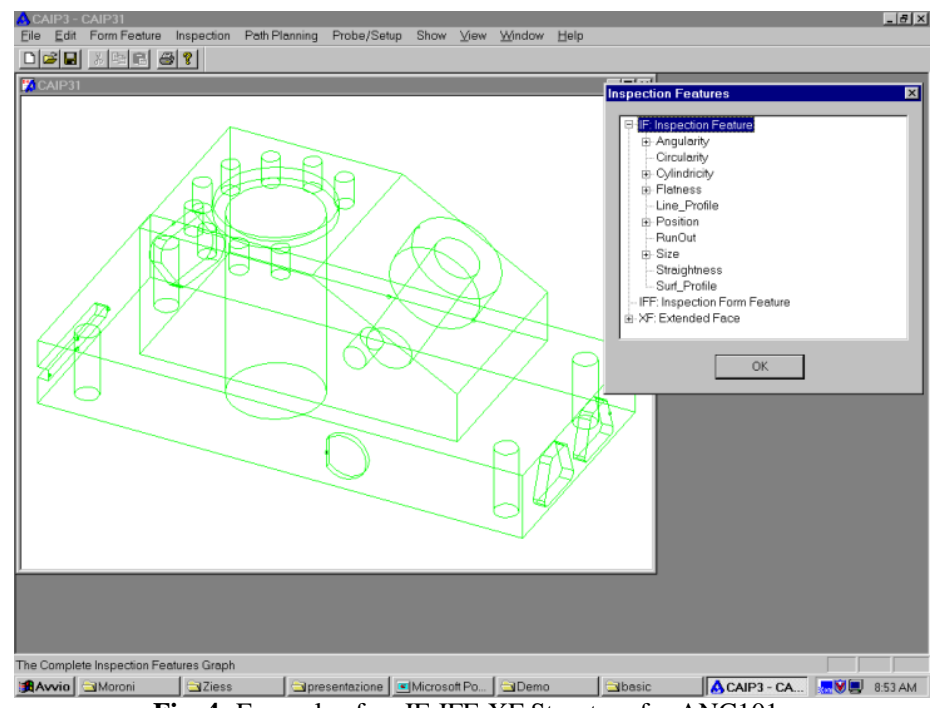

Fig. 4: Example of an IF-IFF-XF Structure for ANC101.

When the part program has been built, a computer simulation or its execution on a CMM permit to report the actual cycle time $t_{c}$. This is an expensive and often useless task, in fact when $t_{c}$ is greater than the imposed $T_{c}$, it is necessary to modify the inspection plan, resulting in a new, often very different, plan. For this reason a hyerarchical approach, making use of more refined $t_{c}$ estimation at each step, has been chosen, so to have a continuous feedback on the choices under consideration during the decisional process.

However, when time constraint $T_{c}$ is not respected $\left(T_{c}<t_{c}\right)$, a reduction of the number of IF to be inspected and of the related measuring points must be operated. An utility score $U$ for each IF, which depends on the accuracy grade of the operations (IT parameter, as defined in the ISO standards) and the capability $\left(\mathrm{C}_{\mathrm{p}}\right)$ of the manufacturing process, has been discussed in [14].

A further feature selection problem concerns the part-related coordinate system. In fact, the alignment procedure is the procedure that finds the existing relationship between the nominal part-related coordinate system and the actual one, and establishes it as the reference frame to be referred to during the inspection. The definition of the alignment procedure is a delicate issue due to the geometrical deviations of the actual part from the nominal one. These deviations are caused by factors such as actual manufacturing processes, CMM accuracy, and sampling strategies. The selection of the most appropriate XFs that will be measured by CMM and used in

\section{Part set-up and probe configuration}

Configuring a probing system means to define its orientation, dimensions and structure. In the probe configuration problem, a set of probes with different orientations and structures should be defined in order to inspect the required faces (XFs) of a part. This implies at first to identify the probe geometric constraints by the analysis of the part and group them in order to find sets of part faces accessible with common constraints. This kind of analysis depends on part set-up. Then, using the previously defined constraints, it implies to configure the more suitable real probe to inspect each set of part faces corresponding to each specific part set-up.

A fixture holds a part in a given spatial configuration (position and orientation) relative to the machine reference frame. This means to define precisely the part location inside the coordinate measuring machine workspace in order to collect and elaborate properly the measuring data. The highly flexible resources availability in the fields of machining, assembly and inspection has carried out to the development of reconfigurable workholding fixtures. Modular fixturing systems, the best solution available to date, are built from standard components and their configurations could be easily modified according to part geometry. The design of a modular fixture should ensure that: the part is not free to move relative to the workspace (kinematic analysis); each part of the batch assumes the same spatial configuration within tolerance; the fixture can withstand forces acting on the part during the inspection.

The fixture and probe design are strongly related. In fact, if it is chosen a unique part orientation, inspecting all the required surfaces of the part may imply to use a large number of probes, by involving a large probe changing time and cost. If it is chosen a set of different part fixtures, it is probably needed to use a unique probe configuration, by involving a large inspection time due to the part set-up changing. Therefore, the optimal trade-off between probe configuration and fixture design, with constraints related to the inspection time and accuracy, has to be found out.

The problem of simultaneous design of part fixtures and probing system for dimensional inspection by CMM is addressed through the steps shown in Figure 5.

Initially, reasoning on part geometric model, the system performs an accessibility analysis. This analysis is based on the concept of form feature: a form feature is a subset of the part boundary that has a meaning with respect to a particular application. In this context we have to find out the possible approach direction and the geometrical constraints for a real 
probing system to measure a face involved in a tolerance relation. This analysis refers to the part reference frame (PRF), thus the accessibility directions are expressed as unit vectors in the PRF.

To be able to cluster the accessibility constraints related to different faces of the part, in order to configure a real even complex probing system, it is necessary to define a spatial configuration of the part in the CMM workspace. Particularly, it is necessary to define the part orientation in the machine reference frame (MRF). Of course, a set of different orientations may be needed to be able to inspect all the required faces of the part. This is the second step of Figure 5, and is a very important step in order to optimize the inspection cycle [15].

Given a part orientation in the MRF and a set of faces to be measured in this setup the system transforms the accessibility constraints to the MRF and performs the constraints clustering [16] and the probing system configuration [17] trying to minimize the time needed to change the probe or to reorient it (if a motorized head is used).

Simultaneously, a fixture configuration able to fix the part in the given orientation has to be found out. It is currently under development a fixture design module able to define the configuration of a CMM dedicated modular fixture. By now only the kinematics analysis has been addressed [18].

The probe and fixture configurations are two very complex tasks, which may require rule based AI techniques. Therefore, in order to reduce the computational time we consider these as two parallel processes, even if they are still partially related. In fact the following situations may arise: the fixture influences the accessibility to a face (XF) to be measured in a given orientation (often); it is not possible to find a feasible fixture to fix the part in a given orientation (possible); it is not possible to find a feasible probe to measure a face (XF) in a given orientation (rare).

In the last two cases a direct feedback to the part orientation module has to be considered. The first situation instead may be solved generating a couple of independent solutions to the two problems and then verifying the complete feasibility. If problems arise the nature of the problems will suggest the possible changes in either probing system configuration or fixture configuration or both.

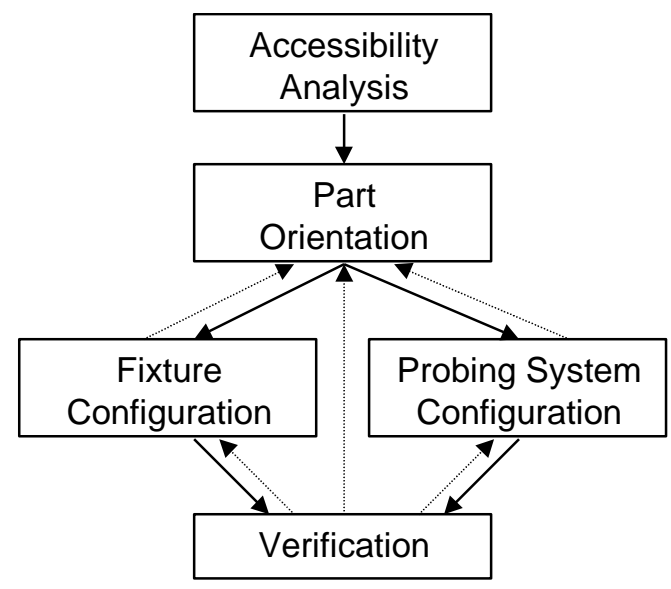

Fig. 5: Fixture and Probing System Configuration.

\subsection{Accessibility analysis}

In literature different solutions to the accessibility problem exist. Corrigall and Bell verifies the accessibility to the part of a defined set of probe configurations through the analysis of probe approach directions generated from a solid model of the component by means of a spatial decomposition technique [19]. Spyridi and Requicha introduce direction cones as a tool for expressing local and global accessibility of surface features [20]. This approach defines only the orientations of the probe as the minimum number of direction cones, which contain the accessibility directions of all the faces to inspect (minimal clustering problem). The minimal clustering problem is NP-complete: an heuristic approach is used [21]. Khoshnevis and Yeh present an evolution of direction cone concept that tries to reduce the computational efforts [22]. Lim and Menq implement a method to determine the suitable orientation of the probe by accessibility analysis [23]. Depince and Bennis define a visibility domain for each point on the surface to inspect and combine the visibility domains [24]. Limaiem and ElMaraghy present a method for feature accessibility analysis where a light source is put on the measuring point and the projected image of the part is defined on a spherical shell (ICSS) centred at the measuring point [25].

The approaches found in literature aim mostly to define the orientation of the probe, while its geometry, its volume and its tip form are almost neglected. This is due to the schematic representation of the probe, generally seen as a line generally adopted in literature or as a simple single stylus probe. An attempt to consider the probe length and volume is shown in [26], even if it considered a simplified probe too.

The proposed accessibility analysis performs reasoning on part geometric model in order to find out the possible approach directions and the geometrical constraints for a real probing system to measure a face (XF) involved in a 
tolerance relation (IF). As previously stated, this analysis is based on the concept of form feature. A taxonomy of form features useful in this context is given in [27]. For each form feature it is possible to find out the accessibility directions and the dimensional constraints for a probe, i.e. the accessibility constraints as shown in blue in Figure 6 . The accessibility constraints for the two case studies are shown in Table 1 and Table 2. The parameters of Tables 1 and 2 describe the maximum diameter of the tip, the minimum effective working length of the stylus, the maximum diameter of the stylus, the maximum and minimum length of the stylus, the maximum diameter of the probe, the whole length of the probe system, the kind of access: parallel or perpendicular to probe axis (axial or radial respectively), the orientation of the probe and of the stylus. In Table 1 and 2 Y means Yes and N means No.

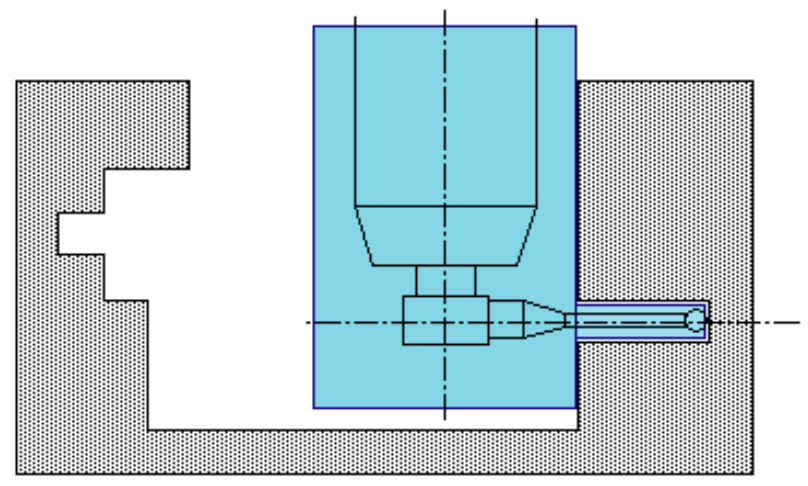

Fig. 6: An Example of Accessibility Constraint.

\subsection{Part orientation}

In the literature great efforts in the fields of computer aided machining fixture design have been done. On the contrary no applications concerning the design of a part fixture in the context of dimensional inspection by coordinate measuring machines may be reported. Spyridi in [4] and Ziemian in [27] have tried to solve the problem by the clustering of accessibility cones computed in different ways. However, they both consider orientations for which no part fixture is required. This means that only stable poses of the part on the CMM table are considered. In [28], instead, a set of part orientations assuming any 3D orientations, which may require fixturing, is determined with heuristics reasoning by clustering directions obtained through a visibility maps based accessibility analysis. The authors, however, do not consider the geometry and the volume of the probe and do not address the fixture design and the probing system configuration.

The part setup optimization problem may be defined as follows: given a set of accessibility constraints, find the number of best part orientations for which all the accessibility constraints will become feasible. In this definition we consider the accessibility direction as the relevant constraint and we define it as feasible if and only if it is possible to orient a probe along that accessibility direction. Therefore, we consider as feasible the accessibility directions which form an angle with the ram axis smaller than $\pi / 2$, where the ram axis is directed as $-\mathrm{Z}$ in the MRF. The best part orientation is defined in terms of inspection uncertainty. Because of the inspection uncertainty may be reduced if we consider a measure in a direction parallel to one of the machine axes, the problem consists in the definition of the transformation for which all the considered accessibility directions become feasible and the number of item that is parallel to the machine axes is maximum.

To solve this optimization problem we consider a two steps approach. In the first one the minimum number of part orientations has to be defined. This means that for each possible part orientation there exists a subset of faces (XFs) whose accessibility directions may become feasible in the same time. Considering all the accessibility directions, it will be possible to orient a part so that all the directions become feasible if and only if all these directions belong to the same planar half-space. Each accessibility direction has been considered applied in the origin of the PRF and the plane through the origin, for which all the points identified by the unit vectors have a distance from the plane characterized by the same sign, has been searched. The minimum number of part orientations has been found clustering all the accessibility directions in the minimum number of subsets characterized by points above or below the identified plane. In the second step, for each subset of faces the best part orientation with respect to the machine reference frame has to be defined. Given the minimum number of part orientations and the accessibility directions that become feasible in each part orientation; the transformation of the accessibility directions from the PRF to the MRF has been carried out by means of the rotation matrix. The best part orientation problem consists in the definition of the transformation for which all the considered accessibility directions become feasible and the number of them that is parallel to the machine axes is maximum. Therefore, the problem consists in finding the components of the rotation matrix which guarantee that no accessibility directions in the MRF has a $+Z$ component and the number of directions parallel to the machine axes is maximum. It has been solved by a non-linear programming optimization approach. Figure 7 shows the orientation of the benchmarks in the two considered case studies. 
Table 1: CADME Accessibility Constraints

\begin{tabular}{|c|c|c|c|c|c|c|c|c|c|c|c|c|c|c|c|}
\hline & XF1 & & XF2 & & XF3 & & & XF4 & & & XF5 & & & XF6 & XF7 \\
\hline Property & 1 & 2 & 1 & 2 & 1 & 2 & 3 & 1 & 2 & 3 & 1 & 2 & 3 & 1 & 1 \\
\hline $\begin{array}{l}\text { max_tip_diameter } \\
{[\mathrm{mm}]}\end{array}$ & 8 & 8 & 11 & 11 & 11 & 11 & 11 & 11 & 11 & 11 & 11 & 11 & 11 & 16 & 16 \\
\hline min_ewl [mm] & 17 & 17 & 11 & 11 & 11 & 90 & 11 & 11 & 90 & 11 & 11 & 11 & 90 & 0 & 0 \\
\hline $\begin{array}{l}\text { max_stylus_dia. } \\
\text { [mm] }\end{array}$ & 10.3 & 1000 & 1000 & 22 & 1000 & 1000 & 1000 & 1000 & 1000 & 1000 & 1000 & 1000 & 1000 & 1000 & 1000 \\
\hline min_length [mm] & 0 & 0 & 0 & 28 & 0 & 0 & 0 & 0 & 0 & 0 & 0 & 0 & 0 & 0 & 0 \\
\hline max_length [mm] & 1000 & 1000 & 1000 & 1000 & 1000 & 1000 & 1000 & 1000 & 1000 & 1000 & 1000 & 1000 & 1000 & 1000 & 1000 \\
\hline $\begin{array}{l}\text { max_probe_dia. } \\
\text { [mm] }\end{array}$ & 1000 & 1000 & 1000 & 1000 & 1000 & 1000 & 1000 & 1000 & 1000 & 1000 & 1000 & 1000 & 1000 & 1000 & 1000 \\
\hline tot_length [mm] & 1000 & 1000 & 1000 & 1000 & 1000 & 1000 & 1000 & 1000 & 1000 & 1000 & 1000 & 1000 & 1000 & 1000 & 1000 \\
\hline radial_access & Y & Y & Y & $\mathrm{Y}$ & $\mathrm{Y}$ & $\mathrm{Y}$ & $\mathrm{N}$ & $\mathrm{N}$ & $\mathrm{Y}$ & $\mathrm{Y}$ & $\mathrm{N}$ & Y & $\mathrm{Y}$ & $\mathrm{N}$ & $\mathrm{N}$ \\
\hline axial_access & $\mathrm{N}$ & $\mathrm{N}$ & $\mathrm{N}$ & $\mathrm{N}$ & $\mathrm{N}$ & $\mathrm{N}$ & $\mathrm{Y}$ & $\mathrm{Y}$ & $\mathrm{N}$ & $\mathrm{N}$ & $\mathrm{Y}$ & $\mathrm{N}$ & NO & $\mathrm{Y}$ & $\mathrm{Y}$ \\
\hline dir_p & 0 & 0 & 0 & 0 & 0 & -1 & 0 & 0 & -1 & 0 & 0 & 0 & -1 & 0 & 1 \\
\hline dir_p & 0 & 0 & 0 & 0 & 0 & 0 & -1 & 1 & 0 & 0 & 0 & -1 & 0 & -1 & 0 \\
\hline dir_probe_z & -1 & 1 & 1 & -1 & -1 & 0 & 0 & 0 & 0 & -1 & -1 & 0 & 0 & 0 & 0 \\
\hline
\end{tabular}

Table 2: ANC101 Accessibility Constraints

\begin{tabular}{llllllllllllllll}
\hline Property & XF9 & XF9 & XF10 & XF11 & XF12 & XF13 & XF14 & XF15 & XF16 & XF17 & XF18 & XF19 & XF20 & XF21 \\
\hline max_tip_dia. & 9.6 & 48 & 8 & 18.48 & 6.4 & 18 & 4 & 3.18 & 12.7 & 6.35 & 50.8 & 7 & 6 & 63 \\
[mm] & 15.9 & 0 & 6.4 & 25.2 & 63.5 & 0 & 6.4 & 3.2 & 38 & 6.4 & 107.7 & 0 & 0 & 0 \\
min_ewl [mm] & 15 & & & & $\infty$ & $\infty$ & $\infty$ & $\infty$ & $\infty$ & $\infty$ & 63.5 & $\infty$ & $\infty$ & $\infty$ \\
max_stylus_d & $\infty$ & $\infty$ & $\infty$ & $\infty$ & $\infty$ & $\infty$ & & & 0 & 0 & 114.3 & 0 & 0 & 0 \\
[mm] & & & & 0 & 0 & 0 & 0 & 0 & 0 & $\infty$ & $\infty$ & $\infty$ & $\infty$ & $\infty$ \\
min_length [mm] & 0 & 0 & 0 & $\infty$ & $\infty$ & $\infty$ & $\infty$ & $\infty$ & $\infty$ & $\infty$ & & & \\
max_length [mm] & $\infty$ & $\infty$ & $\infty$ & $\infty$ & & & & & & & \\
max_probe_d & $\infty$ & $\infty$ & $\infty$ & $\infty$ & $\infty$ & $\infty$ & $\infty$ & $\infty$ & $\infty$ & $\infty$ & $\infty$ & $\infty$ & $\infty$ & $\infty$ \\
[mm] & & & & & & & & & & $\infty$ & $\infty$ & $\infty$ & $\infty$ & $\infty$ \\
Tot_length [mm] & $\infty$ & $\infty$ & $\infty$ & $\infty$ & $\infty$ & $\infty$ & $\infty$ & $\infty$ & $\infty$ & & \\
Radial_access & $\mathrm{Y}$ & $\mathrm{N}$ & $\mathrm{N}$ & $\mathrm{N}$ & $\mathrm{Y}$ & $\mathrm{N}$ & $\mathrm{N}$ & $\mathrm{Y}$ & $\mathrm{Y}$ & $\mathrm{Y}$ & $\mathrm{Y}$ & $\mathrm{N}$ & $\mathrm{N}$ & $\mathrm{N}$ \\
Axial_access & $\mathrm{N}$ & $\mathrm{Y}$ & $\mathrm{Y}$ & $\mathrm{Y}$ & $\mathrm{N}$ & $\mathrm{Y}$ & $\mathrm{Y}$ & $\mathrm{N}$ & $\mathrm{N}$ & $\mathrm{N}$ & $\mathrm{N}$ & $\mathrm{Y}$ & $\mathrm{Y}$ & $\mathrm{Y}$ \\
dir_probe_X & 0 & 0 & -1 & 0.5 & 0 & 1 & 1 & 0 & 0 & 0 & 0 & -1 & 0 & 0 \\
dir_probe_y & 0 & 0 & 0 & 0 & -1 & 0 & 0 & -1 & 0 & 0 & 0 & 0 & -1 & 0 \\
dir_probe_Z & -1 & -1 & 0 & -0.86 & 0 & 0 & 0 & 0 & -1 & 1 & -1 & 0 & 0 & 1 \\
dir_stylus_X & 0 & 0 & -1 & 0.5 & 0 & 1 & 1 & 0 & 0 & 0 & 0 & -1 & 0 & 0 \\
dir_stylus_y & 0 & 0 & 0 & 0 & -1 & 0 & 0 & -1 & 0 & 0 & 0 & 0 & -1 & 0 \\
dir_stylus_z & -1 & -1 & 0 & -0.86 & 0 & 0 & 0 & 0 & -1 & 1 & -1 & 0 & 0 & 1 \\
\hline
\end{tabular}

(A)

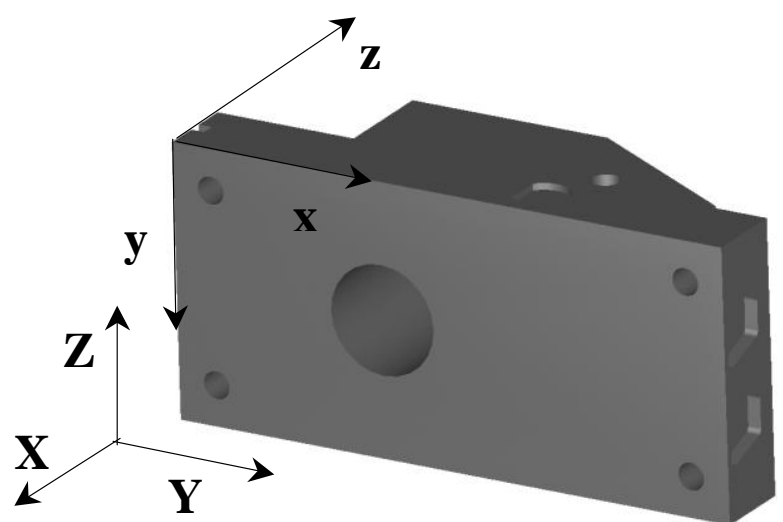

(B)

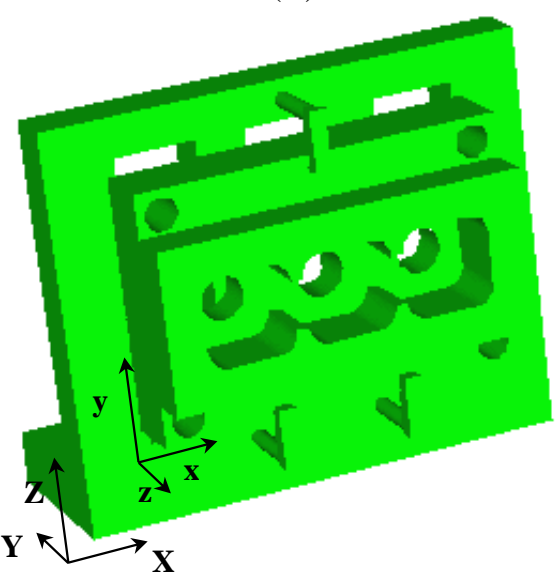

Fig. 7: Best Part Orientation for Both the Case Studies: A) CADME; B) ANC101. 


\subsection{Probing system configuration}

In the literature very little work has been developed concerning the configuration of a probing system. Dodini et al. present a method to generate probe constraints that work on an approximate boundary representation of the workpiece [29]. A different solution has been proposed by Herman in [30], where he considers combinations of particular form features that bind the accessible directions, the tip and the stylus dimensions for each single probe element, and then the probe configuration. However, this method needs the definition of a particular instance of probe configuration for each classified feature. Therefore, it is not general and not suitable for complex part generated by multiple intersections of form features.

The proposed strategy requires two stages. In the first one a clustering determined which XFs can be inspected by the same probe and the problem is to minimize the probe number. The second stage defined the probe configurations more suitable to inspect all the XFs accessible with a given set of constraints identified during the clustering stage.

The clustering module, starting from a combinatorial comparison among the constraints identified during the accessibility analysis, decides which XFs may be inspected by the same single element probe and which elements can be grouped together in an even complex probe by means of the two strategies: depth-first and $\mathrm{A}^{*}$. These strategies implement the procedures and the behavior of the operators involved in the probe configuration: from the analysis of the probe head need, to the identification of the probe axis directions which are recoverable with a head rotation, and from the combination of the dimensional constraints, to the merging of the compound configurations.

The probe configuration is a complex problem due to the high number of variables that must be considered. The proposed method decomposes the complex problem of probe configuration into sub-problems characterized by a reduced number of decision-making variables. Those sub-problems constitute the sequential steps of the assembly that, beginning from the elementary components of the probe, such as the styli, the probes and so on, create the intermediary components (such as the stylus or the probe systems) coming to the configuration of the probe.

The proposed approach addresses toward a rapid configuration satisfying geometric and technologic criterions. The geometric criterions, due to the accessibility clusters, guide the preliminary choice of the probe elements, while the technological criterions address the assembly of the components in the final probes: they are concerned with the stylus and the probe.

As concerns the stylus, its length and its weight generate an axial stress and a bending moment on the probe. These two stresses are limited by the mechanical properties of the probe. Moreover, the stylus inertia, straight proportional to the stylus mass, may invalidate the measurements of the point coordinate. In fact, higher is the bending moment, higher is the probability that probe acceleration causes false trigger. Therefore, the threshold of the trigger is a variable to choose the stylus. The human experts generally agree that the performances of the probe decrease when the stylus overcomes $20 \mathrm{~mm}$ length, due to the previous exposed reasons. It is advisable to have a probing system, constituted by the stylus and its accessories, as short as possible.

It is assumed as general condition that, if the stylus has a length higher than the maximum value imposed by the corresponding geometric constraint, this difference of lengths is used to reduce the distance between the probing system and the probe head. In other terms, the position of the probe inside the accessibility cluster is not fixed, but it depends on the length of the stylus arranging along an axial direction. Moreover, it is a good rule considering no more than two accessories for the stylus. Each accessory, in fact, introduces uncertainty in the measuring system. Finally, it is possible to define the value of the tip diameter under which the automatic configuration does not choose in order to avoid the measurement of the surface micro-geometry. The probe and its accessories are the stiffest and the heaviest components of the probe. It is better to connect the probe directly to the probing system but they should have the same thread; otherwise an adapter is needed. It is a good rule to avoid adapter holding up a stylus characterized by a thread greater than those of probe. The opposite condition is admitted, but not advisable. The bending moment due to the probe system during the rotation of the probe head is sustained by the drive torque of the probe head. It is a function of the weight and the barycentre position of the probe system. The number of probe accessories is minimized in order to decrease the bending moment of the probe. Moreover, the complexity of the probing system is a further aspect to take into consideration. All the considerations on the assembly uncertainty and the weight limits remain valid, but a further problem of balancing should be taken into account too. An asymmetrical distribution of the masses on the four radial branches of the star moves the barycentre far from the probe from its axis. It introduces a torque on the probe head reducing the accuracy of the measurement and wearing out the joint between the probe and the stylus system. It is a good rule of thumb to reduce unbalanced loads on the probe.

The original developed method follows two ways of reasoning to configure the probe, called fast and exhaustive respectively. Both ways combine the components of the probe coming up to a first acceptable configuration. The fast way implements a depth-first search algorithm. It combines at first only the components more suitable in terms of stiffness (and therefore characterized by the least values of length). If the first choice does not give any acceptable solution, the number of components to consider for assembly is gradually increased up to an exhaustive combination. It aims to give a quick solution, running along the solution that looks like the most suitable. The exhaustive way examines and combines the whole set of components. Once an acceptable configuration has been found, both ways aim to quickly converge towards the remaining three or four best configurations. They introduce some rules to cut off the remaining assembly to effect. Four heuristics have been developed. Two rules provide to reduce the weight and the bending 
moment acting on the probe head, in order to guarantee high dynamic performances. The third heuristic aims to balance the probe. The fourth heuristic reduces the number of components to stabilize structurally the probe; it has a consequence on the uncertainty of the probe. Therefore, the four heuristics translate the requirements of lightness, compactness and stiffness previously defined.

For example to inspect the two benchmarks placed how shown in Figure 7, the cluster of geometrical constraints needed is reported in Table 3, whereas the cylinder connected with the single stylus parallel to the probe axis is called 'axial1', the up to four other cylinders connected with the styli whose axes make a Cartesian coordinate frame with the probe axis are called 'radial1',..., 'radial 4' and the cylinder connected with the probe is called 'axial2'. A picture of the six cylinders of a star cluster is shown in Figure 8. The parameters shown in Table 3 are intuitable, aside from ewl parameter that stands for effective working length, i.e. the minimum length of the stylus to avoid collisions with the part. Each cylinder is characterized by a unit vector (axis direction), a height and a diameter. These cylinders represent the maximum volume inside which the probe can move without colliding with the part when inspecting a form feature of the set. It has been developed a clustering module that generates, for each fixturing of each part of the mix, the set of global constraints, in terms of touch probe orientations and dimensions, to inspect the set of faces accessible in that particular set-up. It starts from the accessibility constraints. The developed clustering module is a knowledge-based system that reproduces, as a sequence of rules, the $\mathrm{A}^{*}$ search in a graph represented problem. This state space representation translates the behaviours implemented by the human beings involved in the probe configuration, "the experts". Therefore, the more suitable geometrical constraints are defined on the base of heuristics considerations. This system has been implemented on a PC using Nexpert ${ }^{\circledR}$, an object oriented expert system development environment, which runs as a Window application. All the details are reported in [16].

\begin{tabular}{|c|c|c|c|c|c|c|}
\hline & axial1 & radial1 & radial2 & radial3 & radial4 & axial2 \\
\hline max_tip_diameter [mm] & 3.18 & 9.6 & 4 & 6.35 & 7 & \\
\hline min_ewl [mm] & 63.5 & 107.65 & 6.35 & 17 & 90 & \\
\hline max_stylus_diameter [mm] & 1000 & 22 & 1000 & 1000 & 1000 & \\
\hline min_length [mm] & 63.5 & 114.3 & 6.35 & 17 & 90 & \\
\hline max_length [mm] & 1000 & 1000 & 1000 & 1000 & 1000 & \\
\hline max_probe_diameter $[\mathrm{mm}]$ & & & & & & 1000 \\
\hline tot_length $[\mathrm{mm}]$ & & & & & & 1000 \\
\hline probehead & & & & & & FALSE \\
\hline axial_access & FALSE & FALSE & TRUE & FALSE & TRUE & \\
\hline radial_access & TRUE & TRUE & FALSE & TRUE & FALSE & \\
\hline
\end{tabular}

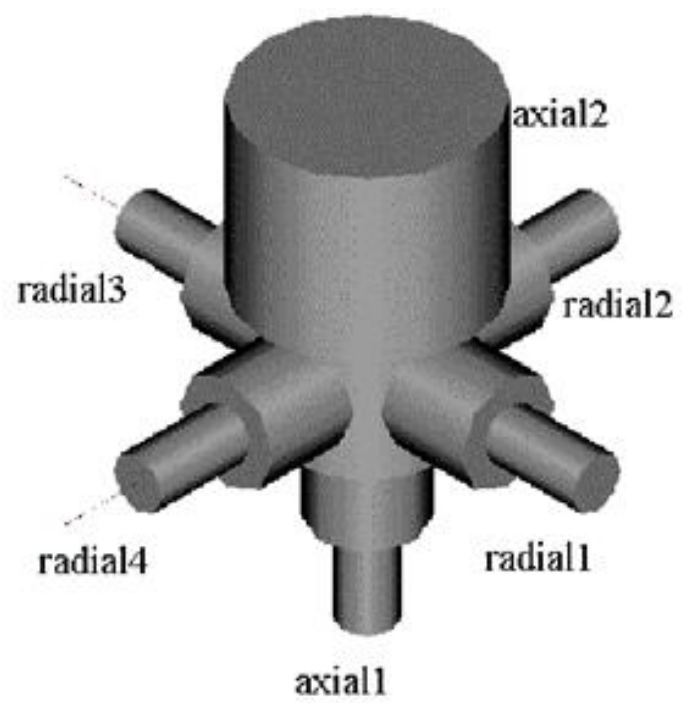

Fig. 8: Star Cluster of Geometrical Constraints.

A further knowledge based system reproduces the procedures and the behaviors implemented by those involved in the probe configuration as a sequence of rules in order to define the touch probe most suitable to inspect all the form features accessible with a given set of constraints, identified during the clustering stage. It is composed of five sets of related rules (knowledge islands); each of them allows the configuration of an element of the probe system. A database, taking into account all the components of a touch probe - probe head, probe extension, probe, stylus adapter, five-way stylus centre, stylus extension and stylus, is considered in order to configure the probe. Here, only a set of Renishaw elements are considered. The probing systems required to inspect both the considered parts are shown in Table 4 and 
Figure 9. Table 4 reports the Renishaw code of the components that constitute the two alternative probing systems. Figure 9 shows the first obtained probing system. All further details are reported in [17].

Table 4: Real Probes for Star Cluster

\begin{tabular}{|c|c|c|}
\hline STAR ELEMENTS] & STAR 1 & STAR 2 \\
\hline axial1_element & $\begin{array}{l}\text { Cylinder_tip_PS_A_5003_1258 } \\
\text { extension SE_A_5003_2280 }\end{array}$ & $\begin{array}{l}\text { Cylinder_tip_PS_A_5003_1258 } \\
\text { extension SE_A_5003_2280 }\end{array}$ \\
\hline axial2_element & Probe_TP2_6WAY & Probe_TP2_6WAY \\
\hline centre_element & $\mathrm{SC} 2$ & $\mathrm{SC} 2$ \\
\hline mass $[\mathrm{g}]$ & 26.9 & 26.9 \\
\hline mass_stylus $[\mathrm{g}]$ & 8.9 & 8.9 \\
\hline radial1_element & $\begin{array}{l}\text { Ball_tip_PS_A_5001_2285 extension } \\
\text { SE_A_5003_2282 }\end{array}$ & $\begin{array}{l}\text { Ball_tip_PS_A_5003_2285 extension } \\
\text { SE_A_5003_2282 }\end{array}$ \\
\hline radial2_element & Ball_tip_PS26R & Bll_tip_PS8R \\
\hline radial3_element & $\begin{array}{l}\text { Ball_tip_PS_A_5003_2285 extension } \\
\text { SE_A_5003_2282 }\end{array}$ & $\begin{array}{l}\text { Ball_tip_PS_A_5003_2285 extension } \\
\text { SE_A_5003_2282 }\end{array}$ \\
\hline radial4_element & Ball_tip_PS26R & Ball_tip_PS8R \\
\hline ranking & 1 & 2 \\
\hline stylus_thread & M2 & M2 \\
\hline stylus_torque $[\mathrm{mm} 2 * \mathrm{~g} 2]$ & 27755.56 & 27755.56 \\
\hline stylus_thread & M2 & M2 \\
\hline
\end{tabular}

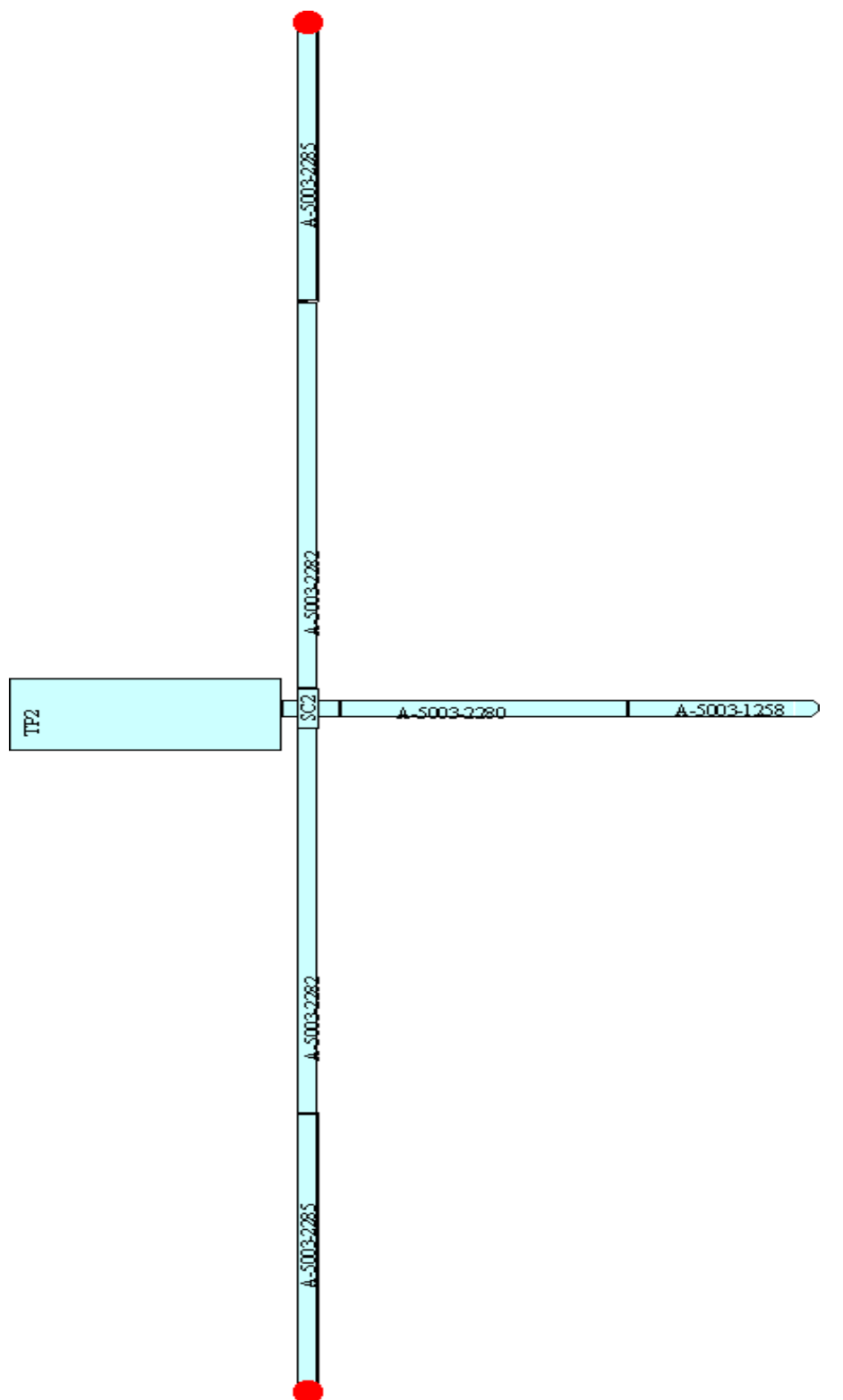

Fig. 9: First Obtained Probe System to Inspect CADME and ANC101 (Called STAR 1 Probe) 


\section{Path planning}

The probe path generation has an impact in improving inspection efficiency and throughput. Once the system has distributed the measuring points on the part, according to an appropriate sampling strategy, the probe path is obtained by connecting all these measuring points with a collision free trajectory.

This path planning problem may be seen as a particular formulation of a traditionally addressed problem: the Travelling Salesman Problem (TSP). It can be represented by a graph, whose nodes are the measuring points and whose arcs connect each couple of points. To each arc a travelling cost is given as the length of the optimal collision free trajectory that connects the two points. The objective function requires a complete circuit with minimum cost, that is all the points have to be measured and the complete path has to be the shortest one.

It is well known that to find an optimal solution to a TSP problem is NP-hard. In fact the complexity of an exhaustive search is $\mathrm{N}$ !, where $\mathrm{N}$ is the number of measuring points in the graph, in the hypothesis of knowing a priori the optimal path connecting each couple of points. Moreover, it is also NP-hard to find the optimal path connecting two points. Therefore, it is almost impossible to have the a priori knowledge needed to solve this TSP problem. The problem is usually solved by finding a promising visiting sequence and, then, calculating a sub-optimal collision free trajectory for the sequence.

Particularly, the two points collision free trajectory generation is mostly addressed considering simple straight probes and polyhedral parts [5], [6], [7], [31]. In [32] and [10] solutions for parts with generic geometry are proposed, but to solve collision problems reconfiguration of the probe and replacement of measuring points are admitted, increasing the complexity of the search.

A different interesting approach to the path planning problem is given in [33], where the sequencing and trajectory problems are solved together creating a simplified network (a roadmap of free space), where the node are measuring points and generated via points and only few arcs are considered to connect measuring and via points with collision free trajectories. The drawbacks are the a priori generation of the additional via points and the choice of a reduced number of possible connecting paths. Still, a single tip probe is considered.

In the CAIP system under development, the path planning module comes after the modules concerning the accessibility analysis, the probe configuration, the set up definition and the distribution of measuring points on the part boundary. Therefore, the main hypotheses are:

1) The measuring points are defined and their replacement is not possible (the distribution follows a predefined sampling strategy);

2) The probe configuration is defined and it can be as complex as possible in the real world (single tip, star, bent, with or without the rotary head);

3) The set-up of the part, the orientation of the probe and the active tip are defined for each measuring point.

Given this context, for each part set up the path planning module has to define the visiting sequence of the points, that the probe is able to measure in the considered set up, and a collision free trajectory for the sequence. Due to the complexity of the problem we are aware that only a sub optimal solution can be found in a reasonable processing time, hence we consider a practical planner. Despite of the interesting approach presented in [33], we prefer to decompose the problem into the sequencing and the trajectory generation problems.

Considering the problem to identify the probe path, for each measuring point it is possible to define a set representing the fuzzy distance between the measuring point and the current position of the probe. If the distance is uncertain and expressed in terms of possibility, the relative range may be represented with a fuzzy sets support range. Moreover the membership function of each fuzzy set will represent the degree with which each value belongs to the fuzzy set possible distances.

It is evident that, due to the complexity in the calculation of the actual inspection point distances, an heuristic algorithm, whose point distances are expressed in terms of possibility ranges, may obtain interesting solutions to the path planning problem. In the proposed case the possibility ranges are approximate estimates of the actual point distances and fuzzy sets theory represents a useful and formal tool in the representation and solution of decision problems characterized by variables defined by means of ranges.

A nearest neighbor search algorithm, based on fuzzy sets theory, has been presented in [34]. The proposed method permit to estimate the distance path as a function of an average difficulty degree met by the probe in the inspection of the part. The difficulty degree is connected with the average actual distance covered by the probe during the shifting between two following measuring points.

The input data are the distances between the current probe position and each residual measuring point. These distances are represented as fuzzy sets. Initially, as general shape of each fuzzy set, a normalized triangle was considered, who's lower and upper limits are respectively equal to the Euclidean distance and to the Manhattan one. The value connected with the membership function equal to one (mode value) corresponds to the mean between Euclidean and Manhattan distances. In [35] we increased the estimate capability of the fuzzy metric, introducing a form feature based fuzzy set estimation. In fact, a difference in the real collision free path length may be detected considering one of these possible situations:

- $\quad$ Couple of points laying in the same face (single XF) ; 
- Couple of points belonging to different faces (XFs) of the same form feature ;

- Couple of point belonging to generic different faces (XFs).

It is important to note that in the CAIP system we already have this kind of information.

Starting from these distances, a fuzzy sets ranking algorithm is applied in order to define the measuring point with minimum fuzzy distance from the current probe position. The ranking operation is performed by means of an algorithm based on the Expected Existence Measure operator.

In [36], a method to generate the complete trajectory given the visiting sequence of the measuring points has been discussed. The problem may be simplified to that of finding out a collision free trajectory for the CMM connecting two measuring points. The idea of a practical planner is common to all the methods presented in literature; however the proposed planner is able to deal with complex probe geometry without any limitation on part geometry. The accessibility analysis guarantees that a trajectory always exists to move the probe from a measuring point to the next one and the planner has to rapidly converge to a sub optimal solution. The proposed planner works in the part space and the part geometric representation is a boundary one.

In order to speed up the search for a collision free trajectory, only polyline paths are generated and two levels of probe approximation are considered. In the first one, we consider only the probe spherical tip and a complete path for the tip is found, as shown in Figure 10a for ANC101 benchmark. Then, the complete probe is considered (with a facet approximation) and the previous path is checked for collision, as shown in Figure 10b for ANC101 benchmark. If collisions are detected a modification of the path is done considering the complete probe. The motivation of this two levels planning is that it is highly probable that two consecutive points in the measuring sequence belong to the same part face (XF) that is accessible by the probe. In these situations it is high the probability that a path connecting the two points which is collision free for the tip is also a collision free path for the complete probing system. Therefore, with the first approximation it is possible to rapidly solve many two points trajectory generations.

(A)

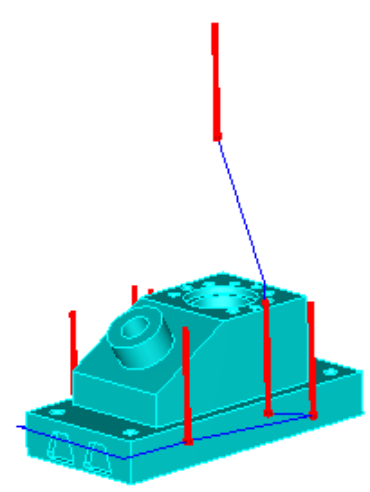

Fig. 10: Path Planning for ANC101: A) Tip Path; B) Complete Probe Path.
(B)

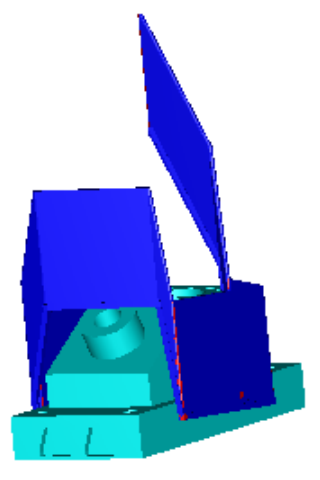

\author{
.
}

.

\section{Conclusions}

A computer aided inspection planning system allows to implement more accurate and efficient CMM inspection procedures. In fact, it allows to automatically plan all the operations concerning with dimensional and geometrical inspection of mechanical parts using optimization and rule based techniques. It gets information from a geometric model of the part, enriched with tolerance specifications (e.g. from a Tolerance Modeler), and makes decision without human intervention. The main decisions concern the selection of the features to inspect, the sampling strategies, the probe configuration, the fixture design and the probe path generation.

Many attempts to achieve the goal of automated inspection planning have been reported in literature. However, a complete planner does not exist, yet. In this paper, we present a complete frame of a CAIP system, able to deal with almost all the decisional stages. Particularly, we discussed original approaches in inspection feature selection, trying to define the right trade-off between inspection accuracy and cost, in part set-up and probe configuration, underling the great importance to consider a conjunct solution, and in path planning, considering the problem of real path estimation. The proposed system may be easily integrated in the product development chain, since it interacts with the design and the manufacturing through Computer Aided Design and Computer Aided Manufacturing platforms.

Surely, the main problems, still open and yet far from a complete solution, are the definition of adequate sampling strategies to inspect the manufactured surfaces of a part, and the evaluation of the consequent contribution to the measurement uncertainty. 


\section{Acknowledgements}

This work has funded partially by the italian M.I.U.R. (Ministry of Instruction, University and Research) and CNR (National Research Council).

\section{References}

[1] H.A. ElMaraghy, W.H. ElMaraghy, "Computer-Aided Inspection Planning (CAIP)" In: J.J. Shah et al., eds. Advances in Feature Based Manufacturing, Elsevier, chapter 16, 1994. http://dx.doi.org/10.1016/B978-0-444-81600-9.50021-3.

[2] A.J. Spyridi, "Automatic generation of high level inspection plans for coordinate measuring machined", PhD thesis, University of Southern California, 1994.

[3] P. Gu, K. Chan, "Generative Inspection Process and Probe Path Planning for Coordinate Measuring Machines", Journal of Manufacturing Systems, Vol.15, No. 4, (1996), pp. 240-255. http://dx.doi.org/10.1016/0278-6125(96)84550-X.

[4] Y.J. Lin, P. Murugappan, "A New Algorithm for Determining a Collision-Free Path for CMM Probe", Int. J. of Machine Tools and Manufacture, Vol. 39, No. 9, (1999), pp. 1397-1408. http://dx.doi.org/10.1016/S0890-6955(99)00006-1.

[5] A. Limaiem, H.A. ElMaraghy, "CATIP: a computer aided tactile inspection planning system", Int. J. Production Research, Vol. 37, No. 2, (1999), pp. 447-465. http://dx.doi.org/10.1080/002075499191869.

[6] V.A. Albuquerque, F.W. Liou, O.R. Mitchell, "Inspection Point Placement and Path Planning Algorithms for Automatic CMM Inspection", International Journal of Computer Integrated Manufacturing, Vol. 13, No. 2, (2000), pp. 107-120. http://dx.doi.org/10.1080/095119200129966.

[7] C.Y. Hwang, C.Y. Tsai, C.A. Chang, 2004. "Efficient inspection planning for coordinate measuring machines", Int J Adv Manuf Technol, Vol. 23, (2004), pp. 732-742. http://dx.doi.org/10.1007/s00170-003-1642-x.

[8] M.W. Cho, H. Lee, G.S. Yoo, J. Choi, "A feature-based inspection planning system for coordinate measuring machines", Int J Adv Manuf Technol, Vol.26, (2005), pp. 1078-1087. http://dx.doi.org/10.1007/s00170-004-2077-8.

[9] F. Zhao, X. Xu, S.Q. Xie, "Computer-Aided Inspection Planning-The state of the art", Computers in Industry, Vol. 60, (2009), pp. 453-466. http://dx.doi.org/10.1016/j.compind.2009.02.002.

[10] Y. Li, W. Wang, H. Li, Y. Ding, 2012. "Feedback method from inspection to process plan based on feature mapping for aircraft structural parts", Robotics and Computer-Integrated Manufacturing, Vol. 28, (2012), pp. 294-302. http://dx.doi.org/10.1016/j.rcim.2011.09.006.

[11] A. Lasemi, D. Xue, P. Gu, P., "A freeform surface manufacturing approach by integration of inspection and tool path generation", Int J Prod Res, Vol. 23, No. 50, (2012), pp. 6709-6725. http://dx.doi.org/10.1080/00207543.2011.618148.

[12] ASME Y14.5, "Mathematical Definition of Dimensioning and Tolerancing Principles", the American Society of Mechanical Engineers, New York, 1994.

[13] G. Moroni, A.A.G. Requicha, "Tolerance modeling and application programming interfaces", Proc. IFIP Symp. On Tools \& Methods for Concurrent Engineering, Budapest, Hungary, May 29-31, 1996

[14] G. Moroni, W Polini, Q. Semeraro, "Part setup and probe design in CMM inspection", CIRP Journal of Manufacturing Systems, Vol. 30, No. $1,(2000)$, pp. 1-10

[15] G. Moroni, W Polini, Q. Semeraro, "The clustering problem in CMM touch probe selection", Proc. of CIRP Int. Seminar on Intelligent Computation in Manufacturing Engineering, Capri, Italy, 1998

[16] G. Moroni, W. Polini, Q. Semeraro, "Knowledge based method for touch probe configuration in an automated inspection system", Int. Journal of Materials Processing Technology, Vol. 76, (1998), pp.153-160. http://dx.doi.org/10.1016/S0924-0136(97)00336-1.

[17] A. Armillotta, L. Carrino, G. Moroni, W. Polini, Q. Semeraro, "An analytical approach to machining deviation due to fixturing", 7th CIRP Int. Seminar on Computer Aided Tolerancing, ENS de Cachan, France, 24-25 April, 2001, pp. 173-182.

[18] M.J. Corrigall, R. Bell, "Probe and component set-up planning for coordinate measuring machines", International Journal of Computer Integrated Manufacturing, Vol. 4, (1991), pp. 34-44. http://dx.doi.org/10.1080/095119291089444775.

[19] A.J. Spyridi, A.A.G. Requicha, "Accessibility analysis for the automatic inspection of mechanical part by coordinate measuring machines", Technical Report No. 257, (1989), South California State University, USA.

[20] A.J. Spyridi, A.A.G. Requicha, S.N. Spitz, "Accessibility analysis for planning of dimensional inspection coordinate measuring machines", Technical Report No. 360, 1998, South California State University, USA

[21] B. Khoshnevis, Z. Yeh, 1993. "An automatic measurement planning system for CMMs", Manufacturing Review, Vol. 6, (1993), pp. $221-227$.

[22] C.P. Lim, C.H. Menq, 1994. "CMM feature accessibility and path generation", International Journal of Production Research, Vol. 32, (1994), pp. 597-618. http://dx.doi.org/10.1080/00207549408956955.

[23] P. Depince, F. Bennis, "Dèfinition de palpeur dédié la mesure de piéces mécaniques", M.M.T. Proceedings of 1st International Conference IDMME '96, 1996, pp. 737-746, Nantes, France.

[24] A. Limaiem, H.A., ElMaraghy, "Integrated accessibility analysis and measurement operations sequencing for CMMs", J. of Manufacturing systems, Vol. 19, No. 2, (2000), pp. 83-93. http://dx.doi.org/10.1016/S0278-6125(00)80002-3.

[25] Y. Wu, S. Liu, G. Zhang, G., 2004. "Improvement of coordinate measuring machine probing accessibility", Precision Engineering, Vol. 28, (2004), pp. 89-94. http://dx.doi.org/10.1016/S0141-6359(03)00080-1.

[26] G. Moroni, W. Polini, "Form Feature Selection in Computer Aided Inspection Planning", Int. Journal of Flexible Automation and Integrated Manufacturing, Vol. 6, No. 1\&2, (1999), pp. 19-41.

[27] C.W. Ziemian, D.J. Medeiros, "Automating probe selection and part setup planning for inspection on a coordinate measuring machine", International Journal of Computer Integrated Manufacturing, Vol. 11, (1998), pp. 448-460. http://dx.doi.org/10.1080/095119298130624.

[28] S. Kweon, D.J. Medeiros, "Part orientation for CMM inspection using dimensioned visibility maps", Computer-Aided Design, Vol. 30, No. 9, (1998), pp. 741-749. http://dx.doi.org/10.1016/S0010-4485(98)00034-7.

[29] A. Dodini, G. Moroni, P. Palezzato, Q. Semeraro, "Touch probe configuration for an automatic inspection system", Manufacturing Review, Vol. 23, (1994), pp. 1-6.

[30] G. Herman, "Volumetric model based programming of co-ordinate measuring machines", In: M.J. Wonzy, J.U. Turner, K. Preiss (Eds.), Geometric Modelling for Product Engineering, Elsevier Science, (1990).

[31] I. Walker, A.F. Wallis, "Application of 3-D Solid Modeling to Coordinate Measuring Inspection", International Journal of Machine Tools \& Manufacturing, Vol. 32, No. 1-2, (1992), pp.195-201. http://dx.doi.org/10.1016/0890-6955(92)90078-U.

[32] H.T. Yau, C.H. Menq, "Automated CMM Path Planning for Dimensional Inspection of Dies and Molds Having Complex Surfaces", International Journal of Machine Tools and Manufacturing, Vol. 35, No. 6, (1995), pp. 861-876. http://dx.doi.org/10.1016/08906955(94)E0024-D. 
[33] S.N. Spitz, A.A.G. Requicha, "Multiple-Goals Path Planning for Coordinate Measuring Machines", IEEE International Conference on Robotics and Automation, San Francisco, California, 2000.

[34] A. Grieco, G. Moroni, F. Nucci, W. Polini, Q. Semeraro, 1999. "Fuzzy Path Planning in CMM Inspection", 4th AITEM Conference, Brescia, Italy, 1999, pp. 107-114.

[35] G. Moroni, W. Polini, M. Rasella, 2001. "Feature based path planning for CMMs", 34th CIRP Int. Seminar on Manufacturing Systems, 16-18 May 2001, Athens, Greece, 2001, pp. 161-167.

[36] G. Moroni, W. Polini, M. Rasella, 2000. "CMM Trajectory Generation", the 33rd CIRP Seminar on Manufacturing Systems, Stockholm, Sweden, 2000, pp. 402-407. 